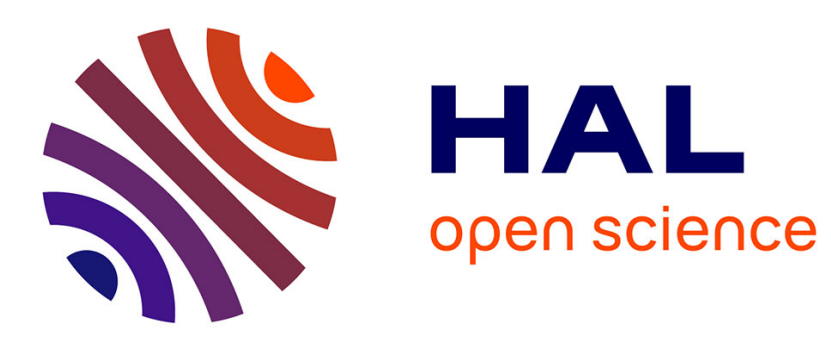

\title{
Cités ouvrières et patrimonialisation : d'un modèle à ses multiples transformations
}

\author{
François Duchêne, Julien Langumier, Christelle Morel Journel
}

\section{To cite this version:}

François Duchêne, Julien Langumier, Christelle Morel Journel. Cités ouvrières et patrimonialisation : d'un modèle à ses multiples transformations. Espaces et sociétés (Paris, France), 2013, Aléas de la patrimonialisation urbaine, 152-153 (1), pp.35-50. 10.3917/esp.152.0035 . hal-01783596

\section{HAL Id: hal-01783596 \\ https://hal.science/hal-01783596}

Submitted on 2 May 2018

HAL is a multi-disciplinary open access archive for the deposit and dissemination of scientific research documents, whether they are published or not. The documents may come from teaching and research institutions in France or abroad, or from public or private research centers.
L'archive ouverte pluridisciplinaire HAL, est destinée au dépôt et à la diffusion de documents scientifiques de niveau recherche, publiés ou non, émanant des établissements d'enseignement et de recherche français ou étrangers, des laboratoires publics ou privés. 


\section{CITÉS OUVRIÈRES ET PATRIMONIALISATION : D'UN MODÈLE À SES MULTIPLES TRANSFORMATIONS}

François Duchêne et al.

ERES I Espaces et sociétés

2013/1 - n० 152-153

pages 35 à 50

ISSN 0014-0481

Article disponible en ligne à l'adresse:

http://www.cairn.info/revue-espaces-et-societes-2013-1-page-35.htm

Pour citer cet article :

Duchêne Françoiset al., "Cités ouvrières et patrimonialisation : d'un modèle à ses multiples transformations », Espaces et sociétés, 2013/1 n 152-153, p. 35-50. DOI : 10.3917/esp.152.0035

Distribution électronique Cairn.info pour ERES.

c) ERES. Tous droits réservés pour tous pays.

La reproduction ou représentation de cet article, notamment par photocopie, n'est autorisée que dans les limites des conditions générales d'utilisation du site ou, le cas échéant, des conditions générales de la licence souscrite par votre établissement. Toute autre reproduction ou représentation, en tout ou partie, sous quelque forme et de quelque manière que ce soit, est interdite sauf accord préalable et écrit de l'éditeur, en dehors des cas prévus par la législation en vigueur en France. II est précisé que son stockage dans une base de données est également interdit. 


\title{
000
}

\section{Cités ouvrières et patrimonialisation : d'un modèle à ses multiples transformations}

\author{
François Duchêne \\ Julien Langumier \\ Christelle Morel Journel
}

Les termes de « cités ouvrières » désignent généralement des ensembles de logements groupés, pavillonnaires ou collectifs, bâtis par une même entreprise pour y loger tout ou partie de son personnel. L'essentiel de cette production $\mathrm{d}$ 'habitat remonte à la fin du $\mathrm{XIX}^{\mathrm{e}}$ siècle et surtout au début du XXe siècle, lorsque le processus d'industrialisation s'affermit dans les pays occidentaux. Les patronats des secteurs industriels traditionnels, confrontés à " la dérobade », arme ouvrière pour mettre en concurrence les employeurs, ont élaboré à tâtons une stratégie pour fixer la main-d'œuvre à proximité de leurs lieux de production

François Duchêne, architecte-urbaniste et docteur en Géographie ; chargé de recherches au laboratoire RIVES de l'ENTPE, membre d'EVS-RIVES (UMR CNRS 5600), université de LyOn.

duchene@entpe.fr

Julien Langumier, docteur en Ethnologie et Ingénieur des travaux publics de l'État; chercheur associé du laboratoire RIVES de l'ENTPE et d'EVS-RIVES (UMR CNRS 5600), Université de Lyon.

julien.langumier@developpement-durable.gouv.fr

Christelle Morel Journel, docteure en Géographie ; maître de conférence à l'Université Jean Monnet de Saint-Étienne, membre d'EVS-Isthme (UMR CNRS 5600).

christelle.morel.journel@univ-st-etienne.fr 
(Noiriel, 2001). Ils ont ainsi adopté un même modèle urbain qui emprunte aux courants de pensée de l'hygiénisme et du paternalisme, plus tardivement à l'architecture des cités-jardins, mais aussi à une généalogie ancrée dans les expérimentations coloniales et esclavagistes. L'expression « cités ouvrières 》 a rapidement désigné cette unique forme de réponse patronale au logement ouvrier, qui s'ordonne souvent en maisons isolées avec jardins. Ce type de production s'est progressivement tari dans la seconde moitié du XXe siècle, avec la prise massive de relais de la construction et de la gestion de logements sociaux par l'État. Toutefois, nombre de ces anciennes cités d'usine marquent encore ponctuellement le paysage des agglomérations et des régions industrialisées, même lorsqu'une urbanisation plus récente les a rejointes.

S'agissant des lieux dépendant de ou ayant été occupés par l'industrie, on constate fréquemment que les projets de patrimonialisation n'émergent qu'après un certain temps, sans doute nécessaire au retrait des principaux protagonistes (Debary, 2002). Or les cités ouvrières constituent un objet hybride - entre habitat et industrie - ce qui leur confère un statut particulier. Bien souvent vendues ${ }^{1}$, les cités ouvrières ne sont plus aujourd'hui gérées par le patronat. Par contre, la population résidente qui a connu ce modèle de gestion ne s'est pas encore totalement renouvelée. Les acteurs ouvriers restent ainsi bien présents, alors que les protagonistes patronaux se sont retirés. De plus, les cités ouvrières continueraient malgré tout de rester des objets porteurs d'un double héritage, entre projet patronal paternaliste et lieu d'appropriation et de sociabilités ouvrières. Ce double visage des cités se traduit aujourd'hui dans des dynamiques de patrimonialisation au sein desquelles se renouvellent les conflits de classes et d'appropriation de l'espace qui se sont joués lors de la vie active de ce modèle urbain de production. Enfin, certaines de ces cités ouvrières - les plus proches des centres-villes - sont aujourd'hui convoitées par de nouveaux groupes sociaux issus des classes moyennes et supérieures, qui ajoutent à l'appropriation et à la valorisation de ce patrimoine un intérêt économique aux enjeux symboliques qu'il recouvrait déjà.

Le devenir patrimonial paradoxal des cités ne tient-il pas dans ces tensions entre la difficile transmission du vécu d'un groupe social et la tentative de valorisation du passé par d'autres à qui elles n'étaient pas initialement destinées, entre l'héritage des pratiques des habitants et la forme des projets urbanistiques, entre un « milieu de mémoire » et un « lieu de mémoire» (Nora, 1984) ? En effet, les cités ouvrières ne sont-elles pas marquées aujourd'hui par la dissolution progressive de la "mémoire vraie réfugiée dans le geste et l'habitude »

1. La vente des cités ouvrières a provoqué des situations différenciées : vendues à leurs occupants et rapidement soumises au libre marché du logement pour les unes (généralement celles les moins dégradées et les plus attrayantes), reprises par des organismes de logements sociaux pour les autres, avec parfois ensuite mise en vente progressive. 
(ibid. : XXIV), sans constituer pour autant des lieux de mémoire, « rescapés d'une mémoire que nous n'habitons plus, mi-officiels et institutionnels, mi-affectifs et sentimentaux » (ibid. : XXV)?

Nous souhaitons analyser ici les tensions qui saisissent les cités autour de projets patrimoniaux plus ou moins constitués. Notre propos s'appuie sur un ensemble d'enquêtes ethnographiques réalisées dans les années 2000 auprès d'habitants de cités ouvrières de la région lyonnaise et stéphanoise ${ }^{2}$. Dans la suite du texte, les encadrés seront utilisés pour faire référence plus précisément à l'une ou l'autre de ces enquêtes. Nous verrons en premier lieu que la question patrimoniale ne touche pas, loin s'en faut, toutes ces anciennes cités. Quand la question y est posée, nous montrerons qu'elle est abordée parfois en référence aux modes d'appropriation des lieux, et d'autres fois au contraire dans la continuité du projet patronal initial. Enfin, dans les cités comme ailleurs, la patrimonialisation peut prendre les chemins d'un conflit de reconnaissance d'un passé ouvrier, contre des aménageurs oublieux et ouverts aux seules classes aisées.

\section{UNE BANALISATION GOMMANT LA SPÉCIFICITÉ OUVRIÈRE DES CITÉS}

Le néologisme patrimonialisation, terme relativement récent, « traduit la volonté d'envisager un processus social de reconnaissance de certains héritages plutôt que les éléments patrimonialisés en tant que tels » (Veschambre, 2007 : 367). Il s'agit bien d'envisager que ce processus puisse suivre d'autres chemins que ceux institués par les services dédiés. Concernant les cités ouvrières, la quantité produite et la diversité des situations font que beaucoup ne sont pas particulièrement investies par une célébration extérieure de leur passé. Si la patrimonialisation est aussi un détour par le passé pour nommer le présent (Rautenberg et al., 2000), on peut se demander si une majorité de cités ouvrières n'a pas disparu ou n'est pas en train de se dissoudre dans d'autres catégories, au risque de ne plus être nommées comme telles.

\footnotetext{
2. Notre propos s'appuie sur une dizaine d'enquêtes menées dans différentes cités ouvrières lyonnaises et stéphanoises, minières, cheminotes ou liées à l'industrie chimique et textile, et récemment publiées dans un ouvrage collectif (Duchêne, 2010). Pour l'essentiel, elles ont été conduites dans le cadre pédagogique d'un enseignement à l'enquête qualitative mené à l'ENTPE. Pour chacune d'entre elles, entre 30 et 50 entretiens semi-directifs, entièrement retranscrits, ont été réalisés auprès d'habitants, pendant les 3 mois que durait chaque enquête. Les entretiens ont été obtenus en faisant du "porte-à-porte » à différents heures de la journée et jours de semaine. Cette méthode d'enquête ne supprime pas totalement le biais observé de rencontres plus aisées avec les personnes les plus disponibles (retraités par exemple) et/ou implantées de longue date dans la cité étudiée. Chaque enquête a été complétée par le recueil d'archives, permettant à chaque fois de mettre en perspective la configuration historique du terrain étudié.
} 


\section{Espaces ouvriers gagnés par l'oubli}

En effet, l'essentiel des logements construits et gérés par une même entreprise l'a été dans une optique très fonctionnelle : sur un plan quantitatif, il s'agissait de pouvoir loger le plus de personnels au plus près de l'activité et de ses rythmes ; sur un plan qualitatif, des travaux ont montré la projection dans l'espace même du logement des hiérarchies sociales existant dans l'entreprise (Frey, 1986). Ce fonctionnalisme calqué sur les besoins et les modes de faire de l'industrie a eu plusieurs conséquences. Les terrains des cités, propriétés des établissements bâtisseurs, ont souvent été acquis à proximité de l'usine, et constituaient des réserves foncières pour les commodités de l'exploitation. D'ailleurs, le contrat d'occupation des locataires, considéré comme un «accessoire au contrat de travail », permettait une révocation rapide des occupants pour les besoins de l'entreprise. Du temps de leur gestion patronale, les cités ouvrières n'avaient donc pas nécessairement de pérennité autre que celle liée aux seuls besoins de l'entreprise. Une autre conséquence de ce fonctionnalisme réside dans la hiérarchie spatiale de ces logements, traduite dans les matériaux employés ou dans les localisations des constructions bâties. En particulier, les foyers de célibataires ou les cités bâties pour les personnels migrants, généralement les moins qualifiés, l'ont souvent été à la hâte avec des matériaux peu durables et sur des terrains des plus résiduels.

Ainsi de nombreuses cités ouvrières, souvent parmi les plus déclassées, ont-elles été détruites. Leur statut de propriété d'une entreprise a facilité juridiquement comme symboliquement leur effacement matériel. C'est le cas par exemple des anciens cantonnements de salariés migrants. Les seules traces qui subsistent de ces logements d'usine sont parfois les écrits produits par leurs anciens habitants, ou des recherches leur redonnant forme et vie (Duchêne et Godard, 2009). De même, certaines cités ont été tronquées de leurs parties les plus déqualifiées, suite à des restructurations d'entreprises nécessitant la disponibilité de nouveaux terrains, l'apport de fonds propres dans le capital ou bien encore une diminution de certaines catégories de personnels. À l'image des démolitions d'îlots insalubres des quartiers anciens qui ont jalonné les politiques urbaines des années 1970, ces disparitions de tout ou partie des cités peuvent être interprétées elles aussi « à la fois comme une forme d'éviction des catégories populaires, mais aussi comme l'effacement symbolique de ces populations dans la mémoire urbaine » (Veschambre, 2005 : 89). C'est le cas par exemple des anciens « chalets » de la cité Maréchal de Saint-Priest, dans la banlieue lyonnaise, au point que cette dernière n'a plus guère de sens et d'existence que pour ceux qui l'ont connue active. 
À proximité de la gare de Saint-Priest, en face de la rue et de l'impasse de l'Industrie, les rues déclinent avec insistance des noms de fleurs. La toponymie ne dessine pas pour autant un quartier clairement identifiable. Quelques maisons identiques des années 1920, aux façades coquettes et aux jardins soignés, s'alignent de manière régulière sur le boulevard des Roses et la rue des Lys, alors que des bâtisses de plus grande taille moins cossues sont édifiées sur les rues des Tulipes, des Résédas et des Chrysanthèmes. Au centre de cet espace, quelques tours des années 1970 se dressent à une dizaine d'étages de hauteur. Ce paysage de faubourg à la périphérie de Lyon ne laisse plus deviner la présence de l'enclave que constituait la cité ouvrière Maréchal jusqu'à la fermeture de l'usine il y a une quarantaine d'années. Enquêter sur cet espace relèverait aujourd'hui de la gageure : «La cité Maréchal, on l'entend de moins en moins. À la limite, c'est le quartier des fleurs, on le verrait plutôt sous cet œil parce que c'est vrai que toutes les rues de la cité Maréchal sont des fleurs. Mais Maréchal..., quand tous les anciens vont partir... [silence] », explique une retraitée de l'usine Maréchal. À la transformation des lieux, s'ajoute la spécificité d'une cité d'ouvrières - du fait de la majorité de femmes employées - qui ne correspond pas toujours aux représentations collectives du logement patronal, fragilisant d'autant plus la pérennité de l'histoire de ce territoire. À l'échelle de la ville de Saint-Priest, ce quartier n'est pas l'objet d'un regard patrimonial comme en témoigne l'ensemble immobilier « Square Maréchal » qui propose une opération standard de logements dans ces anciens espaces industriels devenus pour une part les périphéries résidentielles de Lyon.

En 1924, l'implantation de l'usine Maréchal de tissage et de filage transforme ce bourg agricole en une petite ville industrielle. L'usine produit des tissus pour alimenter l'usine mère de Vénissieux, établie par la famille Maréchal en 1874, qui fabrique des toiles cirées. À la suite de rachats multiples qui conduisent à la fermeture de l'usine en 1965, les maisons en dur sont revendues à leurs occupants alors que les chalets en bois sont démolis pour l'édification de tours d'habitat social.

L'analyse des pratiques mémorielles des anciens « Maréchal » tente de saisir ce que devient l'identité sociale de ce groupe alors que les conditions qui ont contribué à sa constitution ont éclaté, que l'espace qui lui était assigné se recompose, que ses membres disparaissent et que de nouveaux habitants s'installent. La transmission du passé de la cité passe notamment par la demande des nouveaux habitants qui s'approprient une histoire qu'ils n'ont pas vécue pour marquer leur appartenance locale qui accompagne l'accession à la propriété : «On sait qu'avant, la cité c'était derrière, c'était des bungalows en bois. Maréchal a créé son usine. C'était du tissage et il a créé les maisons, ici, pour les ouvriers. En fait c'était des 
... $/ \ldots$

grosses maisons divisées en quatre. [...] C'est Henri Maréchal qui a fait venir tous les immigrés italiens, pour venir travailler dans son usine. On a remarqué qu'il y a pas mal de noms italiens sur les portes... Ça vient de l'époque de Maréchal. » Les discussions avec les voisins ont permis à ce jeune couple de partager cette histoire au prix de quelques reformulations anachroniques, les chalets en bois se transformant en bungalows.

\section{Normalisation d'anciennes enclaves ouvrières}

Un autre phénomène observé lors de ces enquêtes consisterait plutôt en des formes de normalisation de ces enclaves d'habitat ouvrier prises dans les dynamiques contemporaines de leur agglomération. On serait là dans un processus inverse à celui d'une patrimonialisation, dans lequel la cité ouvrière, bien que conservée entière, tendrait à se fondre dans le paysage local au point qu'on ne puisse plus y distinguer de signes d'appartenance à un héritage industriel. C'est le cas par exemple des cités minières des Combes, dans l'agglomération stéphanoise, dans lesquelles on observe des formes de banalisation par les rachats et les transformations successives des maisons.

Au lieu-dit de Montrambert, à cheval sur les communes de La Ricamarie et du Chambon-Feugerolles, la cité des Combes a été construite au début des années 1920 par la société anonyme des Houillères de Montrambert et de la Béraudière afin d'accueillir et de mettre au travail les immigrés polonais de Posdan ou de Katowice qui rejoignaient le bassin houiller de la Loire. La cité était composée de 48 maisons, réparties dans deux combes, à proximité du puits Pigeot, dernier siège d'extraction du bassin de la Loire, fermé depuis 1983. Dès 1973, les Houillères du Bassin du Centre-Midi ont décidé la mise en vente des logements en cités aux ayants droit du régime minier qui, en vertu de l'article 23 du statut du mineur de 1946, bénéficient d'un droit au logement gratuit. Les premières ventes concernent très directement ce public mineur qui acquiert ainsi son premier logement en pleine propriété, en usant de la possibilité du rachat « à vie » de son indemnité de logement voire de la souscription d'un emprunt contracté auprès des Houillères. La maison "manifeste », une dernière fois pourrait-on dire, la générosité de la mine à l'égard de ses ouvriers, cette générosité dont bien des travaux de sciences sociales ont souligné l'ambivalence qui « enferme » les mineurs dans une attitude d'éternelle reconnaissance à la « mine » (Lucas, 1985). 
.......

Après le succès initial, les ventes marquent le pas puis reprennent dès la fin des années 1980 mais il s'agit d'un tout autre public. Les acheteurs ne travaillent plus aux Houillères même si, pour la moitié d'entre eux, ils ont des ascendants directs - père ou grands-parents « des deux côtés » pour un couple - qui ont été mineurs à Montrambert. Le prix de vente des maisons est bien sûr cité comme un critère décisif, mais la valeur tient fondamentalement dans la «tranquillité » de la cité des Combes, son « ambiance familière avec des personnes âgées sympathiques » ou son environnement de « campagne pas très loin des commerces ».

Au milieu des années 2000, une fois passée une période de revente des maisons, les transformations tant sociales que spatiales sont plus importantes. Les chefs de famille d'origine polonaise sont décédés ou partis en maison de retraite. Il n'y a plus de locataires des Houillères, les maisons ont toutes été rachetées. Outre les travaux de réfection qui se poursuivent, un phénomène nouveau a fait son apparition : la destruction complète de maisons pour rebâtir sur un terrain vierge, débarrassé des contraintes liées aux caractéristiques des anciens bâtiments (mûrs en mâchefer, espace restreint, implantation en bordure de terrain, etc.). Il n'est donc plus question des « adaptations » qui ont prévalu aux aménagements des premiers acheteurs (creusement des caves pour créer un deuxième niveau, par exemple) mais de transformations radicales des maisons préexistantes pour approcher au mieux un modèle pavillonnaire plus banal. La banalisation-normalisation de l'habitat, opérée par des personnes dont le positionnement social est un peu plus élevé que celui des ouvriers initiaux, s'est également traduite dans une lettre-pétition adressée au maire du ChambonFeugerolles visant à débaptiser la «cité des Combes » au profit d'une appellation valorisant le site et la situation - les Hauts des Combes - et escamotant le terme de « cité » trop marqué du sceau d'un passé ouvrier et industriel.

La maison acquise auprès de l'ancien employeur constitue plutôt un « patrimoine familial », souvent assez inespéré, conçu comme une opportunité de rejoindre le groupe tant valorisé des propriétaires de maisons individuelles. Fonctionne-t-elle alors comme le « piège » qu'évoque Pierre Bourdieu (1990), en devenant le « lieu de fixation de tous les investissements 》 désormais « bornés à la frontière du seuil, et enfermés dans l'ordre du privé » ? Poser cette question souligne au fond l'insertion progressive des cités dans les circuits économiques banals et les dynamiques des espaces urbains contemporains. 


\section{TRANSMISSION DU VÉCU VERSUS VALORISATION DES LIEUX}

Certaines cités sont cependant saisies par un processus de valorisation, voire de patrimonialisation. Deux idées nourrissent ici la réflexion. D’une part, l'idée de "filiation inversée » développée par J. Davallon pour lequel les contemporains choisissent leurs « pères », le passé étant parfois instrumentalisé afin de servir le présent. D’autre part, l'idée de sélectivité du processus de patrimonialisation, tous les groupes sociaux n'ayant pas un égal accès à la valorisation de leurs espaces, de leurs objets ou de leurs pratiques identitaires (Pinçon et Pinçon-Charlot, 2007). Et les opérations de démolitions successives qui ont touché l'habitat populaire, depuis les grands travaux d'Haussmann jusqu'aux destructions actuelles des grands ensembles, montrent la fragilité des groupes sociaux visés à défendre leur espace de vie et, plus encore, à l'ériger en patrimoine commun de tous. La patrimonialisation des cités ouvrières ne va donc pas de soi. En dépit de la mobilisation de certains habitants et de l'intervention d'acteurs institutionnels, elle suit encore des processus aléatoires, parfois conflictuels.

\section{Revendications d'un entre-soi ouvrier menacé}

Pour les habitants rencontrés, la notion de patrimoine renvoie assez facilement à ce qui appartient au groupe, voire à la famille à travers l'acquisition de la maison. L'observation des interactions entre le modèle et les pratiques montrerait plutôt que les habitants ont inventé leur quotidien malgré un système d'organisation totalisant, mais aussi que les modes d'habiter contribuent aujourd'hui à maintenir présent un fonctionnement dont les tenants et les aboutissants se sont évanouis. C'est-à-dire que, au-delà du logement luimême, les cités sont dépeintes comme un entre-soi où l'interconnaissance et une histoire commune permettent des pratiques de sociabilité supposées inexistantes dans les quartiers plus récents, a fortiori dans les grands ensembles d'habitat social. Il y est aussi souvent question de valeurs communes, de solidarités importées du travail ensemble. De même, la vie associative qui s'y développe est fortement empreinte de pratiques syndicales développées autrefois dans l'usine voisine. Et ce sentiment de bien commun peut être fragilisé par la disparition des anciens ouvriers et l'arrivée de « nouveaux » habitants étrangers à cette histoire. Ainsi ramené aux configurations sociales contemporaines, le patrimoine manifeste bien ici l'idée d'un mouvement de célébration du passé depuis le présent. Pour Gérard Lenclud, la tradition «n'est pas le produit du passé, une œuvre d'un autre âge que les contemporains recevraient passivement mais, selon les termes de Pouillon, un point de vue que les hommes du présent développent sur ce qui les a précédés, une interprétation du passé conduite en fonction de critères rigoureusement contemporains » (1987 : 118). C'est le cas, par exemple, dans d'anciennes cités cheminotes 
collectives de l'agglomération lyonnaise, pour lesquelles l'entrée dans le circuit banalisé du logement social ravive un sentiment identitaire particulièrement vif chez les locataires cheminots.

Construits dans les années 1930 par la compagnie PLM et longtemps gérés par la seule SNCF, les immeubles de faible densité des anciennes cités cheminotes de la région lyonnaise ont été transmis dans les années 1980 à un organisme HLM, la filière Sud-est du groupe Immobilière des Chemins de Fer (ICF), qui les a progressivement réhabilités. ICF est toujours partie prenante du groupe SNCF, et l'entreprise ferroviaire lui verse en retour la quasi-totalité de sa contribution au « $1 \%$ logement ». Ce double lien contribue à renforcer la filiation entre l'entreprise et ses cités d'origine. Cependant, les opérations de réhabilitation, dans les années 1980, ou de rénovation urbaine, dans les années 2000, ont été partiellement financées par des fonds d'État, à la condition de plafonner les loyers des logements rénovés, et d'ouvrir en partie l'accès à ces logements (et aux aides qui les conditionnent), à toutes les personnes répondant aux critères d'accessibilité aux HLM, et non plus aux seuls personnels de la SNCF. Par ce mécanisme, les agents qui logeaient dans ces cités avant leur conventionnement avec l'État ont obtenu le droit d'y rester après leur retraite, ce qui leur était impossible auparavant.

À Oullins, dans la cité cheminote Jacquard, les locataires les plus anciens, encore nettement majoritaires, semblent contester ce glissement de l'entre-soi cheminot vers le «droit commun » du logement social. Ce refus s'y exprime par la construction arbitraire d'une catégorie repoussoir de nouveaux habitants, hâtivement nommés par les anciens les " cas sociaux ». Cette expression fait référence à la réservation d'un contingent de logements par la Préfecture, comme dans tous les HLM, afin que ses services puissent loger les ménages demandeurs en grande difficulté économique. Elle est utilisée par les locataires les plus proches d'un noyau cheminot identitaire, pour exprimer leur attachement à leur corporation et, a contrario, leur rejet de ménages chômeurs qu'ils estiment trop assistés socialement : " Je suis cheminot, je défends un peu mon butin », dit l'un d'eux, retraité. Ainsi la cité constituerait un «butin » accumulé par le fruit de leurs efforts, et qui leur serait progressivement ravi.

À Saint-Priest, dans la cité de la rue Pierre Sémard, l'espace cheminot a été progressivement normalisé à mesure qu'il entrait dans le droit commun des HLM. Ainsi, outre les logements remis aux normes d'habitation, les jardins ouvriers sont devenus pelouses, les garages de fortunes construits à l'aide de traverses de chemin de fer et de tôles ont été démontés, 
... $/ \ldots$

les greniers ont été condamnés, et plus récemment plusieurs immeubles ont été démolis pour être remplacés par des logements sociaux neufs. Le rejet par les plus anciens d'une nouvelle cité aux loyers plus élevés et davantage tournée vers les classes moyennes inférieures, s'exprime ici aussi par une mise à distance dédaigneuse des « extérieurs » ou des «n’importe qui », termes utilisés par les habitants les plus anciens pour qualifier les non-cheminots (Dianoux, 2009).

Derrière ces rejets protéiformes s'exprime aussi la peur d'un déclassement social de ces anciens ouvriers du chemin de fer, dont le statut professionnel tend à ne plus être reconnu comme tel au fur et à mesure des mutations successives du groupe SNCF.

Lorsqu'un groupe social se sent dépossédé d'un bien ou d'une activité fondant son identité ou les moyens de reproduction de sa pérennité en tant que telle, il peut être tenté d'utiliser l'argument patrimonial pour assurer sa survie (Péron, 2001). Émergent alors des velléités de conservation de ces lieux à l'identique, mais l'identique concerne ici avant tout la configuration sociale, même si ses termes s'appuient sur l'espace pour s'exprimer. La patrimonialisation, bien que le terme lui-même soit rarement employé, concernerait dans cette configuration de cités la transmission de valeurs solidaires et d'un vécu dans ces lieux, plutôt qu'une valorisation des lieux seuls, comme vidés de la vie ouvrière qu'ils ont abrités.

\section{Une valorisation par la gentrification des lieux}

Pour les acteurs institutionnels, la patrimonialisation apparaît de manière plus explicite. Elle correspond bien souvent à la sauvegarde de lieux repérés comme remarquables, emblématiques d'une histoire industrielle dont la restitution est le plus souvent pensée dans son volet strictement productif. La place des espaces habités semble moins assurée, même si des inventaires sont élaborés ici ou là, tout comme des zonages de protection. Les inventaires architecturaux singularisent les formes du bâti tandis que le regard des visiteurs extérieurs participe d'une forme d'esthétisation. Le patrimoine relève alors de la reconnaissance par autrui de la singularité du lieu et conduit à une valorisation des cités, initiée par le milieu associatif, encouragée par les municipalités, appuyée par les travaux de recherche. Après la fermeture de l'usine et la disparition de la tutelle paternaliste, ces quartiers font l'objet d'aménagement par les habitants devenus propriétaires. Ils intègrent le marché immobilier en prenant de la valeur. On peut d'ailleurs mesurer cette valorisation à l'intérêt que portent les couches sociales plutôt aisées à cet habitat ouvrier en périphérie des 
centres urbains. C'est le cas, par exemple, dans la cité de la Soie à Décines, où, malgré des découpes mal assurées de propriétés dans ces maisons autrefois occupées par plusieurs familles, les prix de vente ont désormais rejoint voire parfois dépassé le prix local du marché pavillonnaire.

À l'entrée de la commune de Décines, en venant de Lyon, l'avenue Jean Jaurès sépare d'un côté l'ancienne emprise industrielle, d'un ensemble d'habitat semi-individuel, parmi lequel l'ancienne cité ouvrière de la Soie. Construite au milieu des années 1930, cette cité de cinquante maisons et deux immeubles collectifs, d'une école et une église, devait répondre à la volonté du patron de l'usine de soie artificielle de stabiliser ouvriers et ouvrières. En 1958, le départ en retraite du patron fondateur de l'usine entraîne la vente du site à la société Comptoir des textiles artificiels (CTA), qui procède à de nombreux licenciements, avant d'annoncer la fermeture en 1959. Des laboratoires de recherche industrielle ont alors été installés sur le site de l'usine, puis intégrés, avec le patrimoine immobilier que constitue la cité de la Soie, au sein du groupe Rhône-Poulenc Textile. Au début des années 1980, Rhône-Poulenc revend la cité, et les familles de salariés du site, locataires, peuvent acheter les logements qu'elles occupent, lesquels sont au nombre d'environ quatre-vingt, à raison d'un, deux, trois ou quatre logements par maison.

De manière générale, l'arrivée des nouveaux acheteurs correspond à l'entrée dans la cité de ménages au positionnement social légèrement plus élevé que celui des anciens ouvriers, bien qu'appartenant globalement aux mêmes catégories sociales. Ainsi, à travers la différentiation entre « anciens » et « nouveaux », s'exprime la confrontation entre différents modèles et normes de l'habiter, reliés à des aspirations sociales différentes. En particulier, les nouveaux résidents ont été en partie les vecteurs, pour les anciens locataires de la cité, d'un « apprentissage de la propriété », à travers la différenciation des maisons et les stratégies de distinction, considérant leur lieu de résidence comme un patrimoine à entretenir et à faire valoir pour se positionner socialement.

Achetées initialement pour des «bouchées de pain », selon l'expression d'un habitant de la Soie, les maisons et les logements, qui certes ont nécessité ensuite des investissements et de nombreux travaux de réfection, ont vu leur valeur vénale augmenter de façon importante. Ainsi, à travers cette évolution, les habitants savent être propriétaires d'un patrimoine à la valeur élevée, mais avec une situation économique et sociale inchangée. La normalisation de la cité a entraîné la promotion sociale que représente l'accès à la propriété, tout en participant de l'invisibilisation qui touche la 
... $/ \ldots$

classe ouvrière actuellement, ainsi que son histoire. L'arrivée des « autres », de «nouveaux » venus, est donc révélatrice du croisement des parcours sociaux dans la cité, entre continuation de certaines caractéristiques ouvrières, et devenir d'espace pavillonnaire dans une banlieue lyonnaise à dominante résidentielle.

Dans les démarches institutionnelles observées autour des anciennes cités ouvrières, c'est avant tout le bâti qui est désigné. Lorsque le regard patrimonial se tourne vers les cités ouvrières, il s'agit généralement de l'œil de l'architecte intéressé par le modèle pavillonnaire (Debarre, 2007) ou par le lotissement (Dufieux et Garaix, 2007). Dès lors, contrairement à ce que laisse entendre la formule du « tout-patrimoine » (Hartog, 2003), la patrimonialisation ne répond pas aux seules qualités historiques d'un lieu. En effet, « derrière la volonté de mettre en valeur et de conserver certains héritages, certains espaces, ce sont bien des enjeux sociaux et politiques de captation de ressources (symboliques et économiques) et d'appropriation d'espaces qu'il s'agit d'éclairer » (Veschambre, 2007 : 379). Et dans ces configurations, les cités seraient davantage patrimonialisées autour du projet spatial patronal plutôt qu'autour des modes d'appropriation qu'ont inventés, par la suite, leurs occupants. Comme si la figure de l'ouvrier, avec ce qu'elle symbolise encore de résistances collectives et de capacités mobilisatrices, gênait une démarche patrimoniale pour des classes moyennes construites précisément sur la mise à distance sociale des classes populaires.

\section{Patrimonialisation RevendiQuée CONTRe l'efFaCement DU PASSÉ OUVRIER}

En matière de transmission, les aspirations des habitants rencontrés répondent pour une part à la disparition des usines et à la quête de sens qui lui succède, pour une autre part, aux stratégies de valorisation de leurs biens immobiliers et aux aspirations à une autre place dans l'espace social et urbain. Globalement, les actions institutionnelles ou associatives de valorisation du passé industriel suivent autant qu'elles y participent l'installation des couches moyennes et supérieures dans ces quartiers populaires (Bidou-Zachariasen et Poltorak, 2008). Parfois, les échelles s'emboîtent et des trajectoires différentes se dessinent au sein d'une même enclave industrielle : les cités pavillonnaires sont investies et valorisées par les classes moyennes quand les logements collectifs se paupérisent progressivement du fait du vieillissement sur place d'une population de plus en plus modeste, ou par la gestion d'un organisme HLM économe de ses investissements. Et pourtant, les uns et les autres peuvent se 
retrouver dans un combat commun contre une accélération brutale du reclassement de l'ensemble plus large de la zone urbaine. Le recours patrimonial peut alors être utilisé comme un sursaut revendicatif, comme c'est le cas de l'ensemble TASE, à Vaulx-en-Velin.

Construites en 1925 pour loger les personnels de l'usine textile voisine, les maisons et les bâtiments collectifs des cités TASE constituent un ensemble urbain d'une vingtaine d'hectares au sud de Vaulx-en-Velin. Rachetée par le groupe Rhône-Poulenc pour être fermée en 1975, l'usine constitue depuis une importante friche de plusieurs hectares. Avec l'arrivée récente du métro et du tramway à proximité, l'ancien site industriel a été acquis par différents investisseurs immobiliers, qui prévoyaient sa démolition.

Les petites cités ayant été revendues à leurs habitants, et les grandes cités à un organisme HLM, lors de la fermeture de l'usine, chacun des peuplements de l'ensemble TASE poursuit des trajectoires différenciées, dans la prolongation d'une histoire enclenchée du temps de l'activité de l'usine. Pourtant, englobée dans le projet urbain « Carré de soie » porté par le Grand Lyon, la requalification du quartier suscite une même inquiétude des habitants des deux anciennes cités industrielles, qui craignent de devenir les oubliés de ce nouveau quartier promis prochainement aux classes supérieures et aux activités tertiaires.

C'est dans ce contexte que différentes associations des quartiers Sud vaudais créent en 2008 l'association le « Cercle de la soie rayonne » (CSR), avec pour objet « la préservation et la mise en valeur de l'usine TASE, de ses cités et de tous les éléments qui s'y rattachent, et plus généralement de tout patrimoine industriel et de la mémoire ouvrière en rapport avec cette histoire en région Rhône-Alpes » (Berenguer et al., 2009 : 72). Maniant tantôt l'arme du recours juridique contre la révision du Plan local d'urbanisme (PLU) et le permis de démolir l'usine, tantôt la recherche d'alliances avec les milieux intellectuels et patrimoniaux locaux en organisant une contre-proposition urbaine et des colloques à caractère scientifique, le CSR obtient en 2009 l'inscription à l'inventaire supplémentaire des Monuments historiques de la façade du bâtiment principal de l'usine TASE. Depuis, le promoteur immobilier et les collectivités locales ont accédé à une partie des revendications du CSR, en acceptant une réhabilitation des locaux de l'usine et en diminuant de $12 \%$ la surface hors œuvre nette de l'ensemble de logements prévus autour de l'usine. Mais de la création d'un pôle « textisciences » au sein de celle-ci, « reliant le pôle de compétitivité Techtera qui innove dans les textiles techniques avec la tradition de la soie» (ibid., 76), projet porté par le CSR, il n'est encore pas question : la friche réhabilitée devrait accueillir des activités tertiaires banalisées. De même 
.../...

l'idée, revendiquée par le CSR, de créer une Zone de protection du patrimoine architectural, urbain et paysager (ZPPAUP) englobant les cités et l'usine, n'est pas inscrite à l'agenda des collectivités locales. Mais l'un comme l'autre projet souhaitent avant tout témoigner du passé à la fois industriel et ouvrier des lieux et de ses prolongements dans une partie de son peuplement comme dans la tradition industrielle locale.

\section{CONCLusion}

Les modalités de l'émergence du discours patrimonial, ou son absence, ouvrent de nouvelles pistes de recherche quant à sa capacité à reconstruire la cohérence des cités ouvrières. Partant du modèle paternaliste, les enquêtes menées rendent compte à travers les pratiques et les propos des habitants de multiples formes d'adhésions et de résistances, d'appropriations et de détournements de cet habitat patronal qui cristallise aussi accomplissements et frustrations. Elles révèlent bien des pratiques d'habitants qui ne « cessent d'inventer le quotidien » avec « mille manières de braconner » (De Certeau, 1990 [1980]) dans l'ombre des représentations dominantes, homogénéisantes et univoques du modèle. On comprend alors toute la diversité des situations selon la cohésion et l'unité revendiquée des habitants, leur histoire migratoire, leurs appartenances politiques, l'importance ou non des organisations syndicales ou religieuses, la présence ou non d'une école et de commerces, les relations avec l'usine et avec le reste de la commune, les modes d'obtention puis de vente des maisons. Ces éléments constituent chaque fois des ressources spécifiques avec lesquelles les habitants composent, dans le temps, un mode d'habiter irréductible au fonctionnalisme et au rationalisme du modèle d'origine. Les inventaires architecturaux et les périmètres des ZPPAUP recherchent dans la forme et le style du bâti l'archétype de l'habitat patronal alors que la rencontre des habitants témoigne des empreintes laissées et des distances prises avec ce cadre contraignant. Comment, devant le constat d'une telle distorsion, ne pas penser à cette réflexion d'Octave Debary ouvrant sa recherche sur le Creusot en parlant d' ' inflation patrimoniale sans mémoire » (op. cit. : 9) ?

L'entreprise de patrimonialisation qui pourrait saisir rapidement certaines cités ouvrières, à mesure qu'elles seraient investies par des classes moyennes, risque dès lors de recomposer un modèle fictif qui occulterait l'écart entre les attendus des concepteurs et la réalité des pratiques. Ce « retour vers le futur» conduit paradoxalement à un modèle-patrimoine peu attentif au monde social qui l'a précédé, tout comme les cités ouvrières ont elles-mêmes été implantées dans des sociétés rurales considérées comme des terrains vierges et inhabités. Pour Octave Debary, les pratiques ouvrières se transforment en cultures 
ouvrières, dont l'accent mis sur la science, la technique et l'industrie tend à « neutraliser ces domaines, [à] rendre illégitimes les modes traditionnels (politique, syndical) des luttes les ayant pour enjeu, en bref de les 'dépolitiser'. Le paradoxe est à son comble : pour faire l'histoire, les musées de l'industrie doivent sortir de l'histoire " (op. cit. : 154). De même la sauvegarde de Colonies industrielles catalanes des vallées du Llobergat, du Cardener et du Ter, passerait par l'intérêt que des musées scientifiques et techniques locaux portent avant tout à un ensemble productif plus qu'à un mode d'habiter (Dorel-Ferré, 2011). Il s'agit, pour ce qui nous concerne, moins de contribuer à ces entreprises qui consistent à revisiter le passé depuis le présent que d'appréhender ces processus de patrimonialisation comme une permanente tension entre la mémoire et l'oubli, le souvenir et l'occultation, l'histoire du lieu et la grande Histoire, la parole des gens et le discours institutionnel.

\section{RÉFÉRENCES BIBLIOGRAPHIQUES}

Berenguer, J. ; Giannetti, J. ; BÉARD, J. 2009. « Du passé de l'usine tase faisons table mise », L'Archéologie industrielle en France, ${ }^{\circ}$ 54, p. $72-77$.

Bidou-Zachariasen, C. ; Poltorak, J.-F. 2008. « Le 'travail' de gentrification : les transformations sociologiques d'un quartier parisien populaire », Espaces et Sociétés, n 132-133, p. 107-124.

BouRdieu, P. 1990. «Un signe des temps », Actes de la recherche en sciences sociales, $n^{\circ} 81 / 82$, p. 2-5.

De Certeau, M. 1990 [1980]. «L'innommable : mourir », dans L'invention du quotidien 1. Arts de faire, Paris, Éditions Gallimard, p. 276-287.

DEBARRE, A. 2007. «La réhabilitation du pavillonnaire : objet patrimonial et référence architecturale », dans R. de Villanova (sous la dir. de), Conjuguer la ville, Paris, L'Harmattan, p. 217-230.

DeBARY, O. 2002. La fin du Creusot ou l'art d'accommoder les restes, Paris, Éditions du CTHS.

DiAnouX, C. 2009. Entre logement social et logement patronal, un mode d'habiter imprégné par le travail à la SNCF. La cité cheminote de la rue Pierre Sémard à Saint-Priest (69), mémoire du Master recherche Villes et sociétés, Institut d'urbanisme de Lyon, Université Lyon 2.

DOREL-FerRé, G. 2011. « Les colonies industrielles catalanes, un patrimoine exceptionnel mais encombrant», Rives méditerranéennes, $\mathrm{n}^{\circ} 38$.

DuchÊNe, F. ; GodARD, J. 2009. Derrière l'usine, les cantonnements de Salaise-surSanne, Échirolles, Éditions du musée de la Viscose.

DuchÊNE, F. (sous la dir. de) 2010. Cités ouvrières en devenir. Ethnographies d'anciennes enclaves industrielles, Saint-Étienne, PUSE.

Dufieux, P. ; Garaix, L. 2007. Le rêve de la maison. Cités-jardins, lotissements et habitat durable dans le Rhône, Lyon, Éditions du CAUE du Rhône.

FREY, J-P. 1986. La ville industrielle et ses urbanités. La distinction ouvriers/employés, Le Creusot 1870-1930, Bruxelles, Éditions Pierre Mardaga. 
Hartog, F. 2003. Régimes d'historicité. Présentisme et expérience du temps présent, Paris, Seuil.

LENCLUD, G. 1987. « La tradition n'est plus ce qu'elle était... Sur les notions de tradition et de société traditionnelle en ethnologie », Terrain, $\mathrm{n}^{\circ}$ 9, p. 110-123.

LUCAS, P. 1985. La rumeur minière ou le travail retravaillé, Lyon, PUL.

NoIrIEL, G. 2001. Etat, nation et immigration, Paris, Folio histoire.

NorA, P. 1984, « Entre mémoire et histoire », dans P. Nora (sous la dir. de) Les lieux de mémoire, I. La République, Paris, Gallimard, p. VII-XLII.

PÉRON, F. 2001. «Patrimoine culturel et géographie sociale », dans J.-M. Fournier (sous la dir. de) Faire la géographie sociale aujourd'hui, Caen, PUC, p. 19-30.

Pinçon, M. ; Pinçon-Charlot, M. 2007. Les ghettos du gotha. Comment la bourgeoisie défend ses espaces, Paris, Seuil.

Rautenberg, M. ; Micoud, A. ; Bérard, L. ; Marchenay, P. (sous la dir. de) 2000. Campagnes de tous nos désirs. Patrimoines et nouveaux usages sociaux, Paris, Éditions de la Maison des sciences de l'homme.

VeschAmbre, V. 2005. «Le recyclage urbain, entre démolition et patrimonialisation : enjeux d'appropriation symbolique de l'espace. Réflexions à partir de quatre villes de l'Ouest ", Norois, ${ }^{\circ}$ 195, p. 79-92.

Veschambre, V. 2007. «Patrimoine : un objet révélateur des évolutions de la géographie et de sa place dans les sciences sociales ", Annales de géographie, ${ }^{\circ} 656$, p. 361-381. 